Miquel l. y cois.

Rev. Chil. Pediatr. 64 (4); 228-231, 1993

\title{
Hemorragia por várices esofágicas en niños: rendimiento de la escleroterapia
}

\author{
Isabcl Miquel E. ${ }^{1}$; Ling Vargas T. ${ }^{3}$; Eric Saelzer W. ${ }^{1}$; \\ Francisco Ossandon C ?; Juan Ramón Solo H. ${ }^{3}$
}

\section{Hemorrhage from esophageal varices in children: effectiveness of sclerotherapy}

From August 1988 throughoul decomber 1992 nine children with bleeding esophageal varlces were treated by sclercherapy. Portal thrombosis was present in tive cases and chronic liver disease in lhe semaining lour. Five patients had previously been Irealed in other centefs for their bleeding episodes. Introvaricose cyonoacrilate was successtully used in three children with octive blezding. The eradication of esaphageal varices was occomplished in all nine patients wilh $1 \%$ polidocanol weekly injections inlo and oround the voricose vessels. New blecding from esophageal var ices was not obseved along treatment, but one patient bled trom subcardial varices and a gastric devascularization procedure was necessary. No complications that could be attributed to sclerosis of esophageat varices were observed during the period of eradication. Average patient follow up lime was 19 monlhs, range 3 lo 52 months. New bleeding episodes and esophageal slenosis have nol been delected in any patient aicng follow up. Sclerchercpy seems to be a good alternative lorm of treatment in children wilh symplomatic porlal hyperlens on.

IKey words: esophageal and gastric vaices, sclercheıcpy.]

La hemorragia digestiva por várices esofágicas (VE) es relativamente infrecuente en nifos. Su incidencia varía con la coad, la mayoría de los sangramientos ocurre antes de los 10 años, son excepcionales en el primer año de vida'. Su letalidad es de 5 a $9 \%$ en niños con obstrucción de la vena porta y considerablemente mayor en casos con cirrosis hepática de base?

La hipertensión portal se trató clásicamente mediante derivación portosistémica, hasta que el procedimiento se asociara a clevada morbilidad por encefalopatía hepática ${ }^{3-6}$, lo que favoreció el desarrollo de otras opciones de tratamiento como la esclerotcrapia de Jas várices, que se venía realizando desde $1939^{7}$ y se introdujo en 1959 a la pediatría ${ }^{8}$. El objetivo de este trabajo fue estudiar

I. Unidad de Gastroenterología Infantit, Departamento de Pediatría, Facultad de Medicina, Universidad de Chile.

2. Unidad de Cirugía Infantil, Servicio de Pediatría, Hospital San Juan Je Dios. Santiago, Chile.

3. Departamento de Gastroenterología, Servicio de Medicina, Hospinal San Inan de Dios. Santiago, Chile.

Estudio financiado parcialmente por la Fundación Alexander von Humboldt. prospeclivamente, en nuestro medio, los resultados del tralamicnto de las várices esofágicas por esclerosis endoscopica en niños.

\section{Pacientes y Método}

Entre agosto de 1988 y diciembre de 1992, nueve niños ingresaron a la unidad de gastroenterología infantil de nuestro hospital, a causa de hemorragia digestiva debida a várices esofágicas, para rcalizarles escleroterapia. La edad de los pacicntes fluctuaba entre 2 años 6 meses y 15 años. Cinco etan varones. La causa de hipertensión portal era cavernomalosis de la porta en cínco y los cuatro restantes sufrían afecciones crónicas del hígado. La función hepática de estos úlitimos, de acuerdo a Iid clasificación de Child Pugh', era A en dos y B en los orros dos. Los episodios de hemorragia digestiva previos a la incorporación a tratamiento fluctuaban entre 1 y 6 (tabla). Cinco pacientes habían sido tratados previamente por sus hemoragias en otros centros asistenciales. En uno se había realizado derivación portocava, en dos desvascularización csofagogástrica según la técnica de Sugiura y la de Romero-Tones. Los otros dos habían recibido tratamiento incompleto de esclerolcrapia, durante el cual uno había sufrido pleuroneumonía y estenosis esofágica y el otro mediastinitis quimica, quedando ambos con várices residuales, de las que sangraban periódicamente.

Después de jdentıficar las várices como ja causa de los sangramientos, los siñus fucron incorporados al pro- 
grama de escleroterapia. En los procedunicontos sc utilizó habitualmente un endoscopio Olympus 1120 de canal ancho, $y$ en el paciente con estenosis, como excepción, un endoscopio delgado Fujinon LGI-PE El procedimiento sc realizó con anestesia general en los menores de 8 atios; en los mayores se empleó sedación con midazolam y. $\mathrm{cx}$. cepcionalmente, solo anestesia local do la laringc.

Para controlar el sangramienlo activo se inyect $61 \mathrm{cc}$ de cianoacrilato endovaricoso diluido en jipiodol en proporción de 0,5:0,810. La erradicación de las várices esofágicas se hizo mediante inyecciones de polidocanol $1 \%$ endo y paravaricosas, en sesiones semanales, a un centímetro de la unión esofagogástrica. Para la aplicación de polidocanol se utilizó un inyector provisto de una aguja de $5 \mathrm{~mm}$ de largo y $0,5 \mathrm{~mm}$ de diámelio exlerno y pistola de inyec. ción'11.

\section{Resultados}

Tres de nueve pacientes sangraban en el momento de realizar la endoscopia inicial. En todos ellos se logró controlar la hemorragia utilizando cianoacrilato. La erradicación de várices esofágicas se consiguió en los 9 pacientes en 3 a 7 semanas, lo que implicó un número similar de sesiones (promedio 4,25 sesiones y 5 semanas). En el paciente con estenosis csofágica, que no sangraba al ingresar, se eliminó en un primer tiempo, mediante inyección de cianoacrilato, una gruesa várice residual proximal a la estenosis. Inmediatamente después se hicieron dilatacioncs con bujías de Savary Guilliard hasta obtener paso para el endoscopio delgado $c$ inyectar las várices distales a la estrechez. Una vez completada la erradicación de éstas se prosiguió un programa de dilatación del esófago.

Ningún paciente volvió a sangrar por várices esofágicas durante la fase de erradicación, pero uno lo hizo por várices subcardiales después de la sexta sesión de escleroterapia. Esta hemorragia fue detenida mediante desvascularización gástrica, complelándose más tarde la erradicación de várices esofágicas en una sesión adicional de escleroterapia. En el mismo periodo no se registraron grandes escaras, perforación esofágica, ni infecciones en los pacientes. Los pacientes fueron controlados mensualmente durante los tres meses que siguieron a la erradicación y luego cada ures a seis meses. Uno de ellos continuó sus controles en provincia. La duración del seguimiento fluctuó entre tres y 52 (pronedio 19) meses y durante ellos no observamos recidivas de sangramicnto o estenosis csofágica.

\section{Comentario}

La hemorragia digestiva por várices esofágicas es una de las situaciones más graves en pacientes con hipertensión portal. La sobrevida está determinada por la capacidad de cohibir cl sangramiento, la incidencia de recidivas y la intensidad del deterioro de la lunción hepática producida en torno a la hemoragia. La baja incidencia de hemorragia digestiva por esta causa en pediatría ha hecho dificil reunir un número suficiente do casos para evaluar las diversas terapéuticas que se han ensayado, incluida la escleroterapia de las várices. Esta última ha experimentado un notable desarrollar a partir de la década del setenta hasta llegar a ser el tratamiento de elección. Sus resultados varían en diversas comunicaciones y dependen probablemente de sutiles diferencias de procedimiento, así como de la experiencia de los operadores. En nuestro hospital se han tratado nueve niños y 192 adulıos en 56 meses. Cinco de los niños habían vuelto a sangrar después de

Tabla

Caracteres clínicos de nueve niños incorporados a escleroterapia de várices esofágicas entre agosto de 1988 y diciembre de 1992

\begin{tabular}{lcccccccccc}
\hline Paciente & 1 & 2 & 3 & 4 & 5 & 6 & 7 & 8 & 9 \\
Sexo & F & F & M & M & F & M & M & F & M \\
Edad (años) & 2,5 & S & 6 & 3 & 9 & 14 & 15 & 7 & 7 \\
$\begin{array}{l}\text { Episodios hemorragia antes } \\
\text { iniciación de esclerosis de VE }\end{array}$ & 2 & 3 & 6 & 3 & 3 & 6 & 2 & 1 & 2 \\
$\begin{array}{l}\text { Tralamientos previos } \\
\text { Diagndstico }\end{array}$ & si & no & Sí & sí & si & si & no & no & no \\
\hline
\end{tabular}

( $\mathrm{CP}$ = cavernomatosis de la porta, FHC = fibrosis hepática congénita, $\mathrm{CC}$ = cirrosis criptogénica, A VB = atresia de vías biliares, $\mathrm{SBC}=$ sindrome de Budd-Chiari). 
recibir distintas soluciones terapćuticas a su hipertensión portal en otros centros asistenciales, antes de incorporarse al programa de escleroterapia.

En el análisis de los resultados que se comentan, debe considerarse que desde el comienzo se emplearon, hasta en sus detalles mínimos, las tócnicas de escleroterapia descrilas por Soehendra en Hamburgo ${ }^{10}{ }^{11}$ y que además se trabajó en los niños aprovechando la experiencia obtenida en numerosos pacientes adultos.

El control del sangramiento de várices esofágicas mediante escleroterapia se consigue en 80 a $90 \%$ de los $\operatorname{casos}^{12,13}$ y la proporción de buen éxito aumentaría notablemente al usar cianoacrilato en la obliteración de la várice sangrante ${ }^{14}$. La aplicación de esta sustancia requiere familiaridad con los procedimientos convencionales de escleroterapia a fin de aumentar la posibilidad de colocar efectivamentc la substancia en el interior de la várice y evitar daños por adherencia de ésta al endoscopio. En esta experiencia se logró detener la hemorragia en todos los pacientes que sangraban activamente.

La incidencia de sangramientos espontấneos recidivantes alcanza, en adultos, a $30 \%$ en $6 \mathrm{se}$ manas ${ }^{15}$; en nix̃os sometidos a escleroterapia fluctúa entre 0 y 25\%16-18. Ellos se produccn, precozmente, por várices que aún no han sido erradicadas 0 , posteriormente, por escaras sobre várices permeables. Constituyen una complicación grave durante la fase de erradicación, debido a que puede requerirse escleroterapia de urgencia para controlarlos. En el sangramiento por várices gástricas cl manejo con inyección de esclerosante a nivel subcardial es menos efectivo, técnicamente más complejo y probablemente más riesgoso; por estas razones en el paciente en que ocurrió se practicó sólo desvascularización gástrica para contener la hemortagia. Esta altemativa terapéutica permitió cohibir el sangramiento gástrico y completar posteriormente cl programa de esclerosis de várices esofágicas sin abrir el torax.

La hemorragia por várices esofágicas es muy bien tolerada por los pacientes con buena función hepática. En los niños, a diferencia de los adultos, 50 a $66 \%$ de los casos de hipertensión portal son de origen presinusoidal, por cavernomatosis de. la porta, en los que el hígado está habitualmente sanot. Poco más de la mitad de nuesuros pacien- tes cran portadores de cavernomatosis de la porta; el resto tenía algún daño orgánico del hígado de intensidad leve o moderada, lo que debe haber influido favorablemente en los resultados. La ausencia de complicaciones significativas atribuibles al procedimiento y de sangramiento en el scguimiento tardío constituyen hechos favorables a esta modalidad de tratamiento y se comparan positivamente con los resultados obtenidos, por nuestro mismo equipo, en adultos tambićn sometidos a escleroterapia, en quienes la incidencia de sangramiento recidivante fue $19 \%$ en la fase de erradicación con $3 \%$ adicional en el plazo de un año y letalidad de $29 \%$ anual ${ }^{19,20}$. Por cstas razones, pese al reducido número de casos pediálricos reunidos, creemos que la escleroterapia es probablemente, en nuestro madio, un muy buen uratamiento en niños con hemorragia digestiva por várices esofágicas.

\section{Resumen}

Entre agosto de 1988 y dicicmbre de $1992 \mathrm{sc}$ realizó un programa de escleroterapia en 9 niños que habían sangrado por várices esofágicas. La enfermedad base era cavcrnomatosis de la porta en cinco y daño hepático crónico en los otros. Cinco de los pacientes habían sido tratados previamente en relación con sus hemorragias en otros centros. Tres niños que sangraban activamente fueron tratados mediante inyección intravaricosa de cianoacrilato, con resultados positivos. La crradicación de las várices se consiguió en los nueve niños inyectando polidocanol $1 \%$ endo y paravaricoso en sesiones semanales. Ninguno de estos pacientes volvió a sangrar a partir de várices esofăgicas durante la fase de erradicación, aunque uno lo hizo por várices subcardiales. En este caso la hemorragia fue detenida mediante desvascularización gástrica. No se observaron complicaciones atribuibles al procedimiento durante la fase de erradicación. Los pacientes fucron controlados, en promedio, 19 meses (márgenes 3 y 52 meses), durante los cuales no se registraron sangramientos recidivantes y estenosis esofágicas. La escleroterapia de várices esofágicas parecc una buena opción terapéutica en niños portadores de hipertensión portal sintomálica.

(Palabras clave: várices esofágicas y gástricas, cscleroterapia.) 


\section{Referencias}

1. Stevenson RJ: Gastrointestinal bleeding in children. Surg Clin North Am 1985; 65: 1455-1477.

2. Mowat AP: Prevention of variceal bleeding. J Pedialt Gasiroenterol Nulr 1986; 5: 679-687.

3. Conn HO: The rational evaluation and management of portal hypertension. In: Schaffner F, Sherlock S, Lery CM, eds. The liver and its diseases. Jew York: Intercontinental Medical Book Corp. 1974; 289-306.

4. Oldham $K$, Lobe $T$ : Gastrointestival hemorthage in children. Pediatr Clin North Am 1985; 32: 1247-1261.

5. Bernard $O$, Alvarez $F$, Alagille $D$ : Resuluats des anastomoses porto-systemiques dans les cirrhose de l"enfant Arch Fr Pediatr 1985: 42: 249-253.

6. Fonkalsrud E, Myers N, Robinson $M$ : Managentent of extrahepatic portal hypertension in children. Ann Sutg 1974; $180: 487.493$.

7. Crafoord $C$, Frenckner $P$ : New surgical treatment of varicous veins of the esophagus. Acta Otolaryngot 1939; $27: 422-429$

8. Fearon B, Sass-Korisak A. The management of esophageal varices in children by injection of sclerosing agents. Ann Otol Rhinol Laringol 1959; 68: 908-915.

9. Pugh RNH, Murray-Lyon IM. Dawson $L$, es al.. Transection of the esophagus for blecding esophagcal varices. Br J Surg 1973; 60: 646-649.

10. Soehendra N, Nam V, Grimm H, Kempeneers I: Endoscopic obliteration of large esophagogastric varices with bucrylate. Endoscopy 1986; 18:25-26.

11. Sochendra N, Reynders-Frederix U, Doehn M, Buetzow

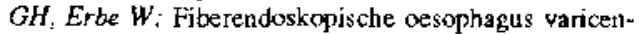
veroedung. Disch Med Wochenschr 1979; 104: 161-163.
12. Paquat K-J, Feussner H: Endoscopic sclerosis and esophagea! balloon tamponade in acute hemorthage from esophagogastric varices: a prospective controlled randomized trial. Hepalology 1985; 5: 580-583.

13. Weslaby $D$, Hayes $P$, Gintson $A$, Folson $R$, Williams $R$ : Controlled clinical trial of injection sclerotherapy for active variccal bleeding Hepatology $1989 ; 9: 274$ 277.

14. Soehendra N, Grimun $H$, Nam V, Brweckner M: 10 Jahres-Erfahrung mit der endoscopischen Sklerotherapie von oesophagogastrischen varicen. Chirurg 1989: 60: $594-598$

15. Graham DY. Smith $J L$ : The course of patients after variceal hemorthage. Gastroenterology 1981: 80: 800 809.

16. Howard $E$, Stringer $M$, Mowat $A$ : Assessment of injection sclerotherapy in the management of 152 children with oesophageal varices. Br J Surg 1988; 75 : 404-408.

17. Spence $R$, Johnston $G$, Odling-Smee $G$, Rodgers $H$ : Bleeding ocsphageal varices with long term follow up Arch Dis Child 1984; 59 : $336 \cdot 340$.

18. Paquet $K$ : Ten years experience with paravariceal injection sclerotherapy of esophageal varices in children J Pediatr Surg 1985; 20: 109-112.

19. Vargas L, Estay R, Ovalle L, Mella B, Soto JR: MorLalidad por hemorragia durante la erradicación de várices esófago-gístricas. XVII Congreso Chileno de Gastroenterología 1991.

20. Ovalle L, Vargas L, Estay R, Mella B, Soto IR: Esclerosis vía crdoscếpica de várices esófago-gástricas: Evaluación a un año. XVIII Congreso Chileno de Gastruenterología 1991 .

\section{AYISO A LOS AUTORES}

Con el objeto de dar prioridad a los trabajos de investigación, en vista de las limitaciones de espacio de la Revista Chilena de Pediatría, el Comité Editorial ha acordado restringir la impresión de casos clínicos a un máximo de dos por cada número. 